STRUCTURAL SCIENCE CRYSTAL ENGINEERING MATERIALS

ISSN 2052-5206

Received 23 April 2021

Accepted 4 August 2021

Edited by J. Lipkowski, Polish Academy of Sciences, Poland

Keywords: nanowires; zincblende structure; diamond structure; analytical descriptions Supporting information: this article has supporting information at journals.iucr.org/b

\section{Analytical description of nanowires. I. Regular cross sections for zincblende and diamond structures. Corrigendum}

\author{
Dirk König $^{\mathrm{a}, \mathrm{b} *}$ and Sean C. Smith
}

antegrated Materials Design Centre (IMDC), UNSW Node, University of New South Wales, NSW 2052, Australia,

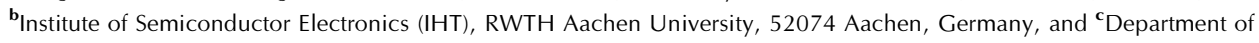
Applied Mathematics, Research School of Physics and Engineering, Australian National University, ACT 2601, Australia. *Correspondence e-mail: solidstatedirk@gmail.com

In the paper by König \& Smith [Acta Cryst. (2019), B75, 788-802], several equations had minor errors in their coefficients defining characteristic lengths and area of zinc-blende nanowire cross sections, thereby deviating from the exact analytical solution by $1.4 \pm 0.8 \%$. A fully corrected version of the paper is provided.

In the paper König \& Smith (2019), several equations had minor errors in their coefficients defining characteristic lengths and area of zinc-blende nanowire cross sections, thereby deviating from the exact analytical solution by $1.4 \pm$ $0.8 \%$. In more detail, such errors occured in equation (8), in the sentence below equation (17), equations (22), (31), (53), (54), (56), (60) to (62), (64), (70), (71), (76) to (79), and in the respective sentence below equations (76) and (77). Additional explanatory text was added under equations (22) and (79). As a follow-on error, some numerical values in Tables 5 and 6 were affected. In Table 5, the height $h$ and area $A$ of the hexagonal nanowire cross section for run index $i=180$ changed slightly (column 2 ), and nearly all values changed in columns 3 to 5 due to a different run index $i$ matching the interface length and height from experiment. These run indices were also changed in the associated text in \$4.1. In Table 6, the cross section area of both nanowire cross sections decreased by $0.6 \%$. The above corrections do not alter any derivations, findings or conclusions of the manuscript. The corrected pdf of this paper is available as supporting information. The authors wish to sincerely apologize for the errors.

\section{References}

König, D. \& Smith, S. C. (2019), Acta Cryst. B75, 788-802.

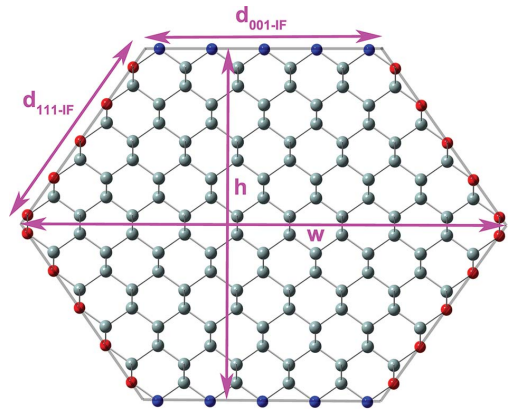

(C) 2021 International Union of Crystallography 